\title{
Basic MBF Blocks Properties and Rank 6 Blocks
}

\author{
Tkachenco V.G. ${ }^{1, *}$, Sinyavsky O.V. ${ }^{2}$ \\ ${ }^{1}$ Institute of Radio, Television, Electronics, Odessa National Academy of Telecommunications, Ukraine \\ ${ }^{2}$ Department of Fundamental Sciences, Odessa Military Academy, Ukraine
}

Copyright $(2017$ by authors, all rights reserved. Authors agree that this article remains permanently open access under the terms of the Creative Commons Attribution License 4.0 International License

\begin{abstract}
Considered all the Monotone Boolean Functions (MBFs) blocks of the sixth rank, it proved a series of new properties MBF blocks. The proposed methods can be used to analyze large ranks MBFs. The tables which describe all of the blocks from 4th to 6th rank are provided. On the basis of these tables are counted typical depending to these blocks.
\end{abstract}

Keywords Monotone Boolean Functions, Inequivalent MBFs, Disjunctive Complement, Conjunctive Complement, Dedekind Number, Types of MBF Blocks

\section{Introduction}

In 1897 R. Dedekind published the article [1], in which he found the number of free distributive lattice elements with four generators. The number of free distributive lattice $\psi(n)$ with $n$ generators coincides with the antichain number in a unit $n$-dimensional cube. In terms of logical algebra, $D(n)=$ $\psi(n)+2-$ is the number of Monotone Boolean Functions (MBFs), depending on $n$ variables $x_{1}, \ldots, x_{n}$. The $\psi(n)$ calculation problem is usually called Dedekind problem. $D$ (0) $-D$ (5) were received by Church in $1940, D$ (6) was calculated by Ward in 1946, $D$ (7) was calculated by Church in 1965 and $D(8)$ was received by Wiedemann [2] in 1991. As it turned out, this problem is rather difficult and it defies solution within the frames of traditional method of generating functions.

However, the method of MBFs classification and analysis based on MBF blocks generation (MBF sets connected by three operations discussed below) has not been considered in literature, excluding the studies [3] and [4], which in a number of cases allows cancelling the MBF search due to the exclusion of all isomorphic blocks and considering only nonisomorphic ones. The MBF blocks analysis allows considering this problem from a new angle.

In [5] the first MBF blocks of various ranks were shown.

The aim of the article is conclusion the basic properties of MBFs and analysis of all the blocks from 4 to 6 ranks.

\section{Results}

Let us recall the main concepts in connection with MBF. Boolean function $f\left(x_{1}, \ldots, x_{n}\right)$ is called monotone, if for any pairs of variables values sets $\left(a_{1}, \ldots, a_{n}\right)$ and $\left(b_{1}, \ldots, b_{n}\right)$, for which the ratio $\left(a_{1}, \ldots, a_{n}\right) \leq\left(b_{1}, \ldots, b_{n}\right)$ is correct, the inequality $f\left(a_{1}, \ldots, a_{n}\right) \leq f\left(b_{1}, \ldots, b_{n}\right)$ is also correct. Among elementary Boolean functions, for example, conjunction and disjunction are monotonic. Any function received from MBFs with the aid of superimposition operation is monotonic by itself. This is why the function generated with the operations of disjunction and conjunction shall be also monotonic.

Say, conjunction $B$ is absorbed by conjunction $A$ if conjunction $B$ contains all variables that are present in conjunction $A$. We shall consider MBF as a Disjunctive Normal Form (DNF), i.e. a sum of products, in which no one is absorbed by the other one. In other words, one set of conjunctions absorbs the other set if all conjunctions of one set are absorbed by the conjunctions of the other set.

We shall designate the Boolean function of $n$ variables ( $n$ rank) $-f_{i}(n)$, here $i-$ is the MBF ordinal number.

We shall use a binary form to present functions. Boolean function $f(n)$ in the form of a bit vector $f(n)=a_{1} a_{2} \ldots a_{t}$, where $t=2^{n}, a_{i}$ can take either the value of 0 or 1 . At that, we shall write the value of the function taken at the lowest set on the right side, while the value taken at the highest set - on the left side. The sets shall be arranged in the following order: the lowest variable $x_{1}$, on the right, the highest variable $x_{n}$ on the left. For example, we shall take a monotonic function from 3 variables $f(3)=x_{1} x_{2}=10001000$. This function equals to 1 only subject to sets $x_{3} x_{2} x_{1}=011$ and $x_{3} x_{2} x_{1}=111$. With other sets the function equals to 0 .

In [6], three unary operations were determined on set of MBFs of any ranks. Duality designated as $\varphi^{-1}$, disjunctive complement designated as $\bar{\varphi}$ and conjunctive complement designated as $\underline{\varphi}$. To obtain disjunctive complement $\overline{f_{i}}(n)$ 
from $i$-MBF $f_{i}(n)$ it is necessary to replace in minimal disjunctive form each conjunction of $m$ variables with conjunction of all $n-m$ variables, beyond original conjunction. To obtain conjunctive complement $\underline{f_{i}}(n)$ from $i$-й MBF, $f_{i}(n)$ of $m$ variables shall be replaced in minimal conjunctive form with disjunction of all $n-m$ variables beyond original disjunction. To obtain dual $\operatorname{MBF} f_{i}^{-1}(n)$ from $i$-й $\mathrm{MBF} f_{i}(n)$ replace all conjunctive operations in minimal disjunctive form with disjunctive operations and simultaneously replace all disjunctive with conjunctive operations. In this case dual MBF $f_{i}^{-1}(n)$ takes a minimal conjunctive form. To obtain dual $\operatorname{MBF} f_{i}^{-1}(n)$ in minimal disjunctive form, remove the parenthesis in the obtained minimal conjunctive form and reduce similar terms.

Definition. MBF block is a set of MBFs completed as regards three operations: duality, disjunctive complement and conjunctive complement, so that any MBF of the block can be obtained from any MBF of the same block by using a certain sequence of these three operations.

For example, for ranks 0 and 1 there exists only one block which consists in the first case of two functions and in the second case - of three functions
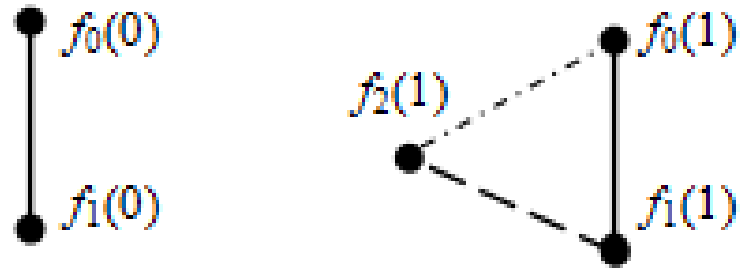

Figure 1. Blocks of zero and first ranks

The dual operation is shown here as a solid graph, disjunctive complement operation - as a dashed line and conjunctive complement operation - as a dash-dot line, $f_{0}(n)$ - zero MBF (equal to 0 with any variables set), $f_{1}(n)$ - unit MBF (equal to 1 with any variables sets), $f_{2}(1)=x_{1}$

Lemma 1. The operation of conjunctive complement is equivalent to a sequence of dual operations, a disjunctive complement and again a dual operation. I.e. in our terms: $\varphi=\varphi^{-1} \bar{\varphi} \varphi^{-1}$

Proof. It is known that dual function $g$ can be obtained by replacing conjunction operations with disjunction operations in initial $f$ operation of conjunction, subject to preserving the operations priority; and vice versa, if the same replacement of operations is carried out in a dual function, we shall obtain the initial function written down in conjunctive normal form (CDF). It follows here from that conjunctive complement of $f$ function and disjunctive complement of $g$ function shall be dual to one another. The lemma is proved.

Consequence. A block can be defined as a set of MBFs closed in relation to three operations: duality, disjunctive complement and conjunctive complement and such that any MBF of the block from any function of the block by using a certain sequence of only two operations $\varphi^{-1}$ and $\bar{\varphi}$.

In this way, a block of functions can be constructed of one function $f$ by using to this function successively the operation of disjunctive complement and then the duality operation. Each function can belong to only one block.

We shall introduce some notions. Block potency is the number of MBFs included. Two blocks are similar if they possess equal potency and provided the MBFs included in them are disregarded, they are identical. Two blocks are isomorphic if any MBF of one block can be obtained from a certain MBF of another block by some variables substitution. By convention, isomorphic blocks are similar.

All MBF blocks can be divided into 4 types. It follows from lemma 1 that all MBFs can be connected with a sequence of two operations - duality and disjunctive complement. The sequence of these two operations can be disconnected or cyclic. In the first case obtain three types of blocks. Blocks of first type is called a block, in which at the ends of the open-loop of sequence are 2 disjunctive self-complementary MBF. Blocks of second type are called a block, in which at one end of the open-loop of sequence are self-dual $\mathrm{MBF}$, and at the other end are disjunctive self-complementary MBF. Block of third type is called a block, in which at the ends of the open-loop of sequence are 2 self-dual MBFs. In second case, self-dual and disjunctive self-complementary MBF no. We get the fourth type of block, which we call cyclic. Examples of MBF blocks are shown below:

1. Containing 2 disjunctive self-complementary MBFs.

Example of such 4 rank block:

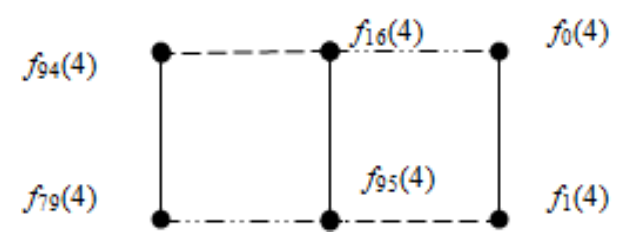

Figure 2. Block containing 2 disjunctive self-complementary MBFs

Here $f_{16}(4)=x_{1} \vee x_{2} \vee x_{3} \vee x_{4}, f_{79}(4)=$ $x_{1} x_{2} \vee x_{1} x_{3} \vee x_{1} x_{4} \vee x_{2} x_{3} \vee x_{2} x_{4} \vee x_{3} x_{4}, f_{94}(4)=$ $x_{1} x_{2} x_{3} \vee x_{1} x_{2} x_{4} \vee x_{1} x_{3} x_{4} \vee x_{2} x_{3} x_{4}, f_{95}(4)=x_{1} x_{2} x_{3} x_{4}$. Functions $f_{0}(4)$ and $f_{79}(4)$ are disjunctive self-complementary.

From this point on the functions are taken from studies [3] and [4].

2. Containing 1 disjunctive self-complementary MBF and 1 self-dual MBF.

Example of such 4 rank block:

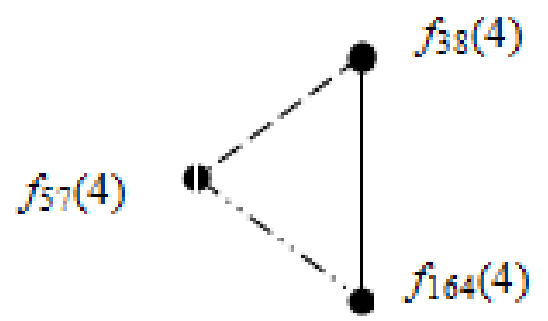

Figure 3. Block containing 1 disjunctive self-complementary MBF and 1 self-dual MBF.

Here $f_{38}(4)=x_{1} x_{2} \vee x_{1} x_{3} \vee x_{1} x_{4}, f_{57}(4)=x_{2} x_{3} \vee x_{2} x_{4} \vee x_{3} x_{4}$, 
$f_{164}(4)=x_{1} \vee x_{2} x_{3} x_{4}$. Function $f_{164}(4)$ disjunctive self-complementary, while $f_{57}(4)$ self-dual.

3. Containing 2 self-dual MBFs.

Example of such 4 rank block:

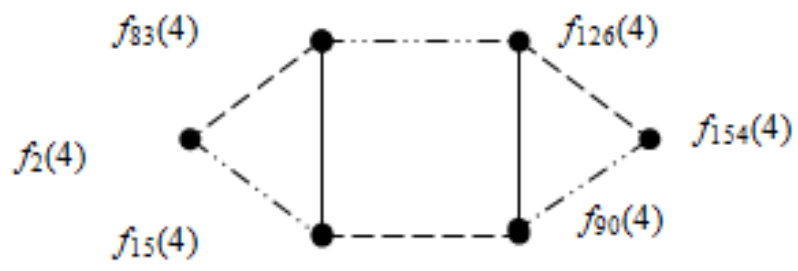

Figure 4. Block containing 2 self-dual MBFs.

Here $f_{2}(4)=x_{1}, f_{83}(4)=x_{2} x_{3} x_{4}, f_{15}(4)=x_{2} \vee x_{3} \vee x_{4}, f_{90}(4)=$ $x_{1} x_{2} x_{3} \vee x_{1} x_{2} x_{4} \vee x_{1} x_{3} x_{4}, f_{126}(4)=x_{1} \vee x_{2} x_{3} \vee x_{2} x_{4} \vee x_{3} x_{4}$ и $f_{154}(4)=x_{1} x_{2} \vee x_{1} x_{3} \vee x_{1} x_{4} \vee x_{2} x_{3} x_{4}$. MBFs $f_{2}(4)$ and $f_{126}(4)$ are self-dual.

\section{Cyclic blocks.}

Example of such 4 rank block:

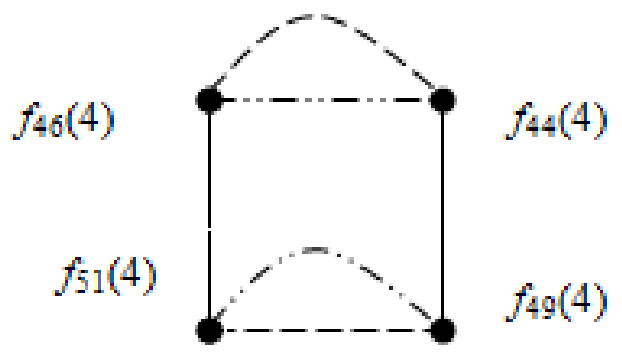

Figure 5. Cyclic block of 4 rank.

Here $f_{46}(4)=x_{1} x_{2} \vee x_{2} x_{3} \vee x_{3} x_{4}, f_{44}(4)=x_{1} x_{2} \vee x_{1} x_{4} \vee x_{3} x_{4}$, $f_{49}(4)=x_{1} x_{3} \vee x_{1} x_{4} \vee x_{2} x_{4}, f_{51}(4)=x_{1} x_{3} \vee x_{2} x_{3} \vee x_{2} x_{4}$. All functions of the block are connected by three operations.

Blocks consisting of two functions can be of two types, consisting of three functions - of one type, consisting of four functions - of three types, consisting of six functions - of three types:

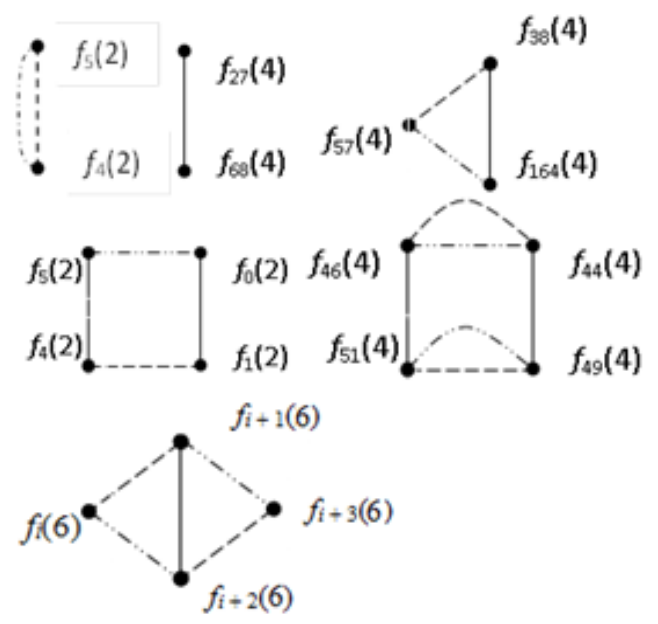

Figure 6. Types of blocks of two, three and four MBFs

Here $f_{i}(6)=x_{1} x_{2} \vee x_{1} x_{3} \vee x_{1} x_{4} \vee x_{1} x_{5} \vee x_{1} x_{6} \vee x_{2} x_{3} x_{4} x_{5} x_{6}$, $f_{i+1}(6)=$

$x_{1} \vee x_{3} x_{4} x_{5} x_{6} \vee x_{2} x_{4} x_{5} x_{6} \vee x_{2} x_{3} x_{5} x_{6} \vee x_{2} x_{3} x_{4} x_{6} \vee x_{2} x_{3} x_{4} x_{5}$, $f_{i+2}(6)=$

$x_{1} x_{2} x_{3} \vee x_{1} x_{2} x_{4} \vee x_{1} x_{2} x_{5} \vee x_{1} x_{2} x_{6} \vee x_{1} x_{3} x_{4} \vee x_{1} x_{3} x_{5} \vee x_{1} x_{3} x_{6} \vee x$ ${ }_{1} x_{4} x_{5} \vee x_{1} x_{4} x_{6} \vee x_{1} x_{5} x_{6}$,

$f_{i+3}(6)=$

$x_{4} x_{5} x_{6} \vee x_{3} x_{5} x_{6} \vee x_{3} x_{4} x_{6} \vee x_{3} x_{4} x_{5} \vee x_{2} x_{5} x_{6} \vee x_{2} x_{4} x_{6} \vee x_{2} x_{4} x_{5} \vee x$ ${ }_{2} x_{3} x_{6} \vee x_{2} x_{3} x_{5} \vee x_{2} x_{3} x_{4}$

Other functions numbers are taken from studies [3] and [4].

The block containing functions $f_{0}(n)$ and $f_{1}(n)$ shall be called base block.

For functions of zero and first rank, there is one block, which is the base (see. Figure 1).

All MBFs of rank of 2 can be presented as 2 blocks, one of them consisting of four MBFs (base) and the other - of two MBFs:
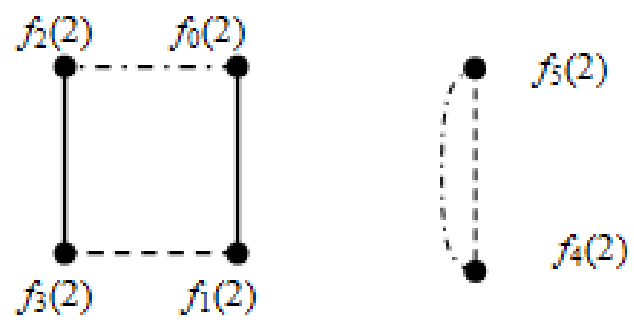

Figure 7. Blocks of rank 2.

Here $f_{2}(2)=x_{1} \vee x_{2}, f_{3}(2)=x_{1} x_{2}, f_{4}(2)=x_{1}, f_{5}(2)=x_{2}$. Function $f_{2}(2)$ shall be disjunctively self-dual, $f_{3}(2)$ conjunctively self-dual, $f_{2}(2), f_{3}(2)$ - self-dual.

Base blocks can be of two types: either the first one, or the second one. To be more precise - either with one self-dual and one disjunctive self-complementary for odd ranks, or with two disjunctive self-dual and one function for even ranks. For example, base blocks of 3 and 4 ranks:

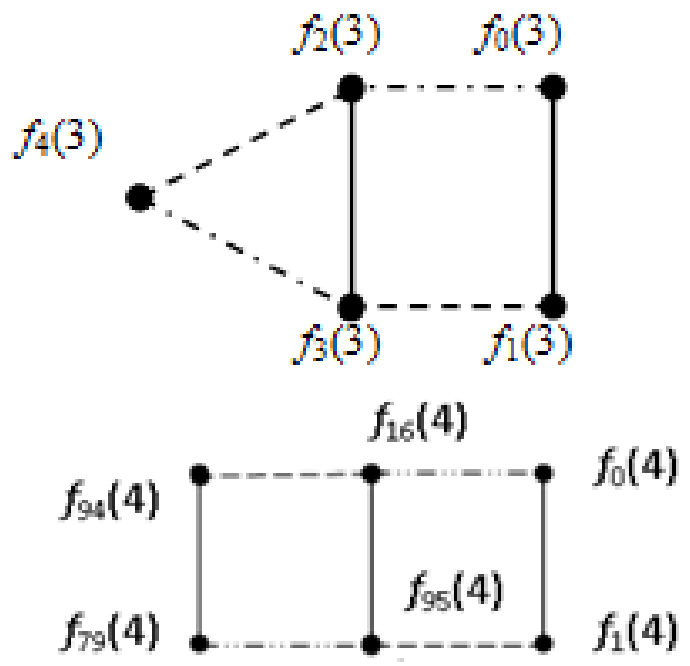

Figure 8. Base blocks of 3 and 4 ranks

Because the base block includes zero and unit MBFs, it is quite simple to show that each function of this block consists of disjunction $C_{n}^{k}$ conjunctions of one length, here $k$-the number of variables in conjunction. This is why the number of base block MBFs of $n$ - rank equals to the number of 
Newton binomial raised to the n-th power factors and additionally zero MBF, i.e. $n+2$.

Suppose MBF is given in the form of DNF: $f\left(x_{1}, \ldots, x_{n}\right)=\bigvee_{j=1}^{k}\left(\delta_{1}^{j} \vee x_{1}\right) \cdot \ldots \cdot\left(\delta_{n}^{j} \vee x_{n}\right)$, here $k-$ the number of conjunctions in DNF, $\delta_{i}^{j}=\left\{\begin{array}{ll}1, & i \notin j \\ 0, & i \in j\end{array}, \quad i-\right.$ variable number, $j$ - conjunction number. By substituting $S=\left(t_{1}, \ldots, t_{n}\right)$ we shall obtain from this function an isomorphic

function

$$
g\left(x_{1}, \ldots, x_{n}\right)=\bigvee_{j=1}^{k}\left(\delta_{t_{1}}^{j} \vee x_{t_{1}}\right) \cdot \ldots \cdot\left(\delta_{t_{n}}^{j} \vee x_{t_{n}}\right) \text {. }
$$

Lemma 2. Functions $f_{1}$ and $g_{1}$ are dual to isomorphic $f$ and $g$ are also isomorphic.

Proof. From MBF $f\left(x_{1}, \ldots, x_{n}\right)$ of any block given in the form of DNF we shall obtain by using dual operation a dual $\operatorname{MBF} f_{1}\left(x_{1}, \ldots, x_{n}\right)=\bigwedge_{j=1}^{k}\left(\left(1-\delta_{1}^{j}\right) x_{1} \vee \ldots \vee\left(1-\delta_{n}^{j}\right) x_{n}\right)$.

Applying $g$ dual operation to isomorphic MBF, we shall obtain dual a MBF $g_{1}\left(x_{1}, \ldots, x_{n}\right)=\bigwedge_{j=1}^{k}\left(\left(1-\delta_{t_{1}}^{j}\right) x_{t_{1}} \vee \ldots \vee\left(1-\delta_{t_{n}}^{j}\right) x_{t_{n}}\right)$, which comes of $f_{1}$ by substituting $S$ and, consequently, it is isomorphic in relation to $f_{1}$. Thus, we have obtained two isomorphic functions $f_{1}$ and $g_{1}$ of two isomorphic functions $f$ and $g$ with the aid of dual operation.

Lemma 3. Functions $f_{2}$ and $g_{2}$ are disjunctive complementary to isomorphic $f$ and $g$ also isomorphic.

Proof. Much as lemma 2. From MBF $f\left(x_{1}, \ldots, x_{n}\right)$ using the disjunctive complement operation we obtain $f_{2}\left(x_{1}, \ldots, x_{n}\right)=\bigvee_{j=1}^{k}\left(\left(\left(1-\delta_{1}^{j}\right) \vee x_{1}\right) \cdot \ldots \cdot\left(\left(1-\delta_{n}^{j}\right) \vee x_{n}\right)\right)$, and from MBF $g\left(x_{1}, \ldots, x_{n}\right)$ we obtain disjunctive complementary $\mathrm{MBF}$ $g_{2}\left(x_{1}, \ldots, x_{n}\right)=\bigvee_{j=1}^{k}\left(\left(\left(1-\delta_{t_{1}}^{j}\right) \vee x_{t_{1}}\right) \cdot \ldots \cdot\left(\left(1-\delta_{t_{n}}^{j}\right) \vee x_{t_{n}}\right)\right) . \quad \mathrm{We}$ can see that MBF can be obtained from $f_{2}$ by substituting $S$ and, consequently it is isomorphic in relation to $f_{2}$

Lemma 4. Suppose function $f$ is given, using some substitution $\alpha$ we shall obtain isomorphic $g$. If we use any succession of three operations: duality, conjunctive and disjunctive complements to these two isomorphic functions, we shall obtain two isomorphic functions $f_{1}$ and $g_{1}$. At that, one of them shall result from the other by substituting $\alpha$.

Proof. Let us remark that the operation of conjunctive complement is equivalent to the sequence of operations: duality, disjunctive complement and again duality (lemma 1). Successively applying to isomorphic MBFs $f$ and $g$ lemmas 2 and 3 we obtain isomorphic $f_{1}$ and $g_{1}$. This shall be more than one function, because each of the three operations is unary and mutually reversible, i.e. an involution, and we cannot obtain different functions out of one function using the same sequence of operations.

From lemmas 2, 3 and 4 it follows immediately that:

Lemma 5. By applying a sequence of three above mentioned operations to any number of isomorphic functions we shall obtain the same number of isomorphic functions.

Theorem 1. Any two MBFs of one block have equal number of isomorphic.

Proof. As a consequence of lemmas we derive that out of two isomorphic MBFs $f$ and $g$ with the aid of any sequence of the three operations described above we also obtain isomorphic MBFs $f_{1}$ and $g_{1}$.

Let us choose arbitrary MBFs $f$ and $f_{1}$ from block 2 . Suppose that $f$ is more isomorphic than $f_{1}$. By the definition of MBF block, $f_{1}$ can be obtained from MBF $f$ by some sequence of operations $P$. Let us apply the same sequence $P$ to all functions isomorphic to $f$. According to the proved above, as the result we shall obtain functions isomorphic to $f_{1}$. Consequently, we have obtained the number of isomorphic the number of isomorphic MBFs to $f_{1}$ equal to the number of isomorphic MBFs to $f$, which contradicts to the presumption. The theorem is proved.

Theorem 2. For each MBF in the block the number of isomorphic MBFs in the block is the same.

Proof. Suppose an MBF of block $f$ has maximal set of isomorphic functions in the block. We shall designate this set through $A$. We shall take any other nonisomorphic to $f$ function $g$. Select the sequence of operations, under which function $f$ turns into function $g$. According to the block definition, such sequence can be always realized. From function $g$ and all its isomorphic we shall obtain a set $B$ functions with the same sequence of operations. All MBFs from the set $B$ by definition of block are in the same block. It follows from lemma 5 that MBFs in set $B$ shall be isomorphic to one another and their number shall be equal to the number in set $A$. The theorem is proved.

The number of isomorphic MBFs for one function in the block shall be called a block index. It follows from the definition of isomorphic blocks and from lemmas 2, 3, 4 and 5 that the statement is correct:

Consequence 1. Isomorphic blocks have identical index.

Consequence 2. Blocks of the first and third type have an index no more than 2. Since the function of self-dual is isomorphic must also be self-dual self-complementary and isomorphic disjunctive must also be self-complementary, and these blocks are only with two of this type of MBF. While blocks of type 2 have index 1 , because they have one self-dual and one disjunctive self-complementary and isomorphic to it in the block does not exist. Blocks of the fourth type may be of any index.

Because for any nonisomorphic MBFs of one block, $f$ and $g$ according to theorem 2 possess equal number of 
isomorphic for each function $f$ and $g$, consequently, the number of isomorphic for any function of the block is the divider of the number of all MBFs in the block. This is why the block index is the divider of the number of all MBFs in the block. The result of the division is the number of nonisomorphic MBFs in the block. It also follows that if we take all isomorphic MBFs to this function, we shall obtain the number of isomorphic blocks.

\section{Conclusions}

With this classification we can define various properties of MBFs.

For example, we shall consider partitioning all MBF blocks of $4^{\text {th }}$ rank according to such classification.

Table 1. Blocks of the $4^{\text {th }}$ rank containing 2 disjunctive self-complementary MBFs:

\begin{tabular}{|c|c|c|c|c|}
\hline Nos & $\begin{array}{c}\text { Number of } \\
\text { MBFs in the } \\
\text { block }\end{array}$ & $\begin{array}{c}\text { Number of } \\
\text { isomorphic } \\
\text { blocks }\end{array}$ & $\begin{array}{c}\text { Block } \\
\text { index }\end{array}$ & $\begin{array}{c}\text { Number of } \\
\text { nonisomorphic } \\
\text { blocks }\end{array}$ \\
\hline$C_{10}$ & $C_{11}$ & $C_{12}$ & $C_{13}$ & $C_{14}$ \\
\hline 1 & 6 & 1 & 1 & 1 \\
\hline 2 & 2 & 3 & 1 & 1 \\
\hline
\end{tabular}

The number of nonisomorphic blocks (or isomorphic blocks groups) of the 4 th rank, i.e. the sum of values of the last columns in all 4 tables $B N(4)=\sum C_{i 4}=7, i=1, \ldots, 4$.

The number of all blocks of rank 4 , i.e. by summing the product of values in the third column by the fifth in all 4 tables $B(4)=\sum C_{i 2} \cdot C_{i 4}=24, i=1, \ldots, 4$.

Table 2. Blocks of the $4^{\text {th }}$ rank containing 1 disjunctive self-complementary MBF and 1 self-dual MBF:

\begin{tabular}{|c|c|c|c|c|}
\hline Nos & $\begin{array}{c}\text { Number of } \\
\text { MBFs in the } \\
\text { block }\end{array}$ & $\begin{array}{c}\text { Number of } \\
\text { isomorphic } \\
\text { blocks }\end{array}$ & $\begin{array}{c}\text { Block } \\
\text { index }\end{array}$ & $\begin{array}{c}\text { Number of } \\
\text { nonisomorphic } \\
\text { blocks }\end{array}$ \\
\hline$C_{20}$ & $C_{21}$ & $C_{22}$ & $C_{23}$ & $C_{24}$ \\
\hline 1 & 3 & 4 & 1 & 1 \\
\hline
\end{tabular}

Table 3. Blocks of the 4 th rank, containing 2 self-dual MBFs:

\begin{tabular}{|c|c|c|c|c|}
\hline Nos & $\begin{array}{c}\text { Number of } \\
\text { MBFs in the } \\
\text { block }\end{array}$ & $\begin{array}{c}\text { Number of } \\
\text { isomorphic } \\
\text { blocks }\end{array}$ & $\begin{array}{c}\text { Block } \\
\text { index }\end{array}$ & $\begin{array}{c}\text { Number of } \\
\text { nonisomorphic } \\
\text { blocks }\end{array}$ \\
\hline$C_{30}$ & $C_{31}$ & $C_{32}$ & $C_{33}$ & $C_{34}$ \\
\hline 1 & 6 & 4 & 1 & 1 \\
\hline
\end{tabular}

Table 4. Cyclic blocks of the 4 th rank:

\begin{tabular}{|c|c|c|c|c|}
\hline Nos & $\begin{array}{c}\text { Number of } \\
\text { MBFs in the } \\
\text { block }\end{array}$ & $\begin{array}{c}\text { Number of } \\
\text { isomorphic } \\
\text { blocks }\end{array}$ & $\begin{array}{c}\text { Block } \\
\text { index }\end{array}$ & $\begin{array}{c}\text { Number of } \\
\text { nonisomorphic } \\
\text { blocks }\end{array}$ \\
\hline$C_{40}$ & $C_{41}$ & $C_{42}$ & $C_{43}$ & $C_{44}$ \\
\hline 1 & 4 & 3 & 4 & 1 \\
\hline 2 & 12 & 3 & 2 & 1 \\
\hline 3 & 12 & 6 & 2 & 1 \\
\hline
\end{tabular}

The number of inequivalent MBFs of the 4th rank [7]
$R(4)=\sum \frac{C_{i 2} \cdot C_{i 4}}{C_{i 3}}=30, i=1, \ldots, 4$ (The number of MBFs in the block to be divided by the block index and multiplied by the number of nonisomorphic blocks, then summarized in all tables).

The number of all MBFs of the 4th rank (Dedekind number) $D(4)=\sum C_{i 1} \cdot C_{i 2} \cdot C_{i 4}=168, i=1, \ldots, 4$ Number of nonisomorphic disjunctive self-complementary MBFs of the 4th rank $D C N(4)=\sum\left(2 C_{14}+C_{24}\right)=5, i=1, \ldots, 4$

The number of all disjunctive self-complementary MBFs of the 4th rank $D C(4)=\sum\left(2 C_{14} \cdot C_{12}+C_{24} \cdot C_{22}\right)=12$, $i=1, \ldots, 4$

The number of nonisomorphic self-dual MBFs of the 4th $\operatorname{rank} \operatorname{DSN}(4)=\sum\left(2 C_{34}+C_{24}\right)=3, i=1, \ldots, 4$

The number of all self-dual MBFs of the 4th $\operatorname{rank} D S(4)=\sum\left(2 C_{34} \cdot C_{32}+C_{24} \cdot C_{22}\right)=12, i=1, \ldots, 4$

The number of nonisomorphic completely non-symmetrical MBFs of the 4th rank (only the lines where $\mathrm{C}_{42} \mathrm{C}_{43}=4 !=24$.) $R N(4)=\sum \frac{C_{i 1} \cdot C_{i 4}}{C_{i 3}}=0, i=1, \ldots, 4$ are to be summarized.

The number of completely non-symmetrical MBFs of the 4th rank (only the lines where $\mathrm{C}_{42} \mathrm{C}_{43}=4$ ! = 24.) $N S(4)=\sum C_{i 1} \cdot C_{i 2} \cdot C_{i 4}=0, \quad i=1, \ldots, 4 \quad$ are to be summarized

The same for the 5 th rank.

Table 5. Blocks of the 5th rank containing 1 disjunctive complementary $\mathrm{MBF}$ and 1 self-dual MBF:

\begin{tabular}{|c|c|c|c|c|}
\hline Nos & $\begin{array}{c}\text { Number of } \\
\text { MBFs in the } \\
\text { block }\end{array}$ & $\begin{array}{c}\text { Number of } \\
\text { isomorphic } \\
\text { blocks }\end{array}$ & $\begin{array}{c}\text { Block } \\
\text { index }\end{array}$ & $\begin{array}{c}\text { Number of } \\
\text { nonisomorphic } \\
\text { blocks }\end{array}$ \\
\hline$C_{20}$ & $C_{21}$ & $C_{22}$ & $C_{23}$ & $C_{24}$ \\
\hline 1 & 7 & 1 & 1 & 1 \\
\hline 2 & 7 & 5 & 1 & 2 \\
\hline 3 & 7 & 10 & 1 & 2 \\
\hline 4 & 7 & 20 & 1 & 1 \\
\hline 5 & 7 & 30 & 1 & 1 \\
\hline
\end{tabular}

Table 6. Cyclic blocks of the 5 th rank:

\begin{tabular}{|c|c|c|c|c|}
\hline Nos & $\begin{array}{c}\text { Number of } \\
\text { MBFs in the } \\
\text { block }\end{array}$ & $\begin{array}{c}\text { Number of } \\
\text { isomorphic } \\
\text { blocks }\end{array}$ & $\begin{array}{c}\text { Block } \\
\text { index }\end{array}$ & $\begin{array}{c}\text { Number of } \\
\text { nonisomorphic } \\
\text { blocks }\end{array}$ \\
\hline$C_{40}$ & $C_{41}$ & $C_{42}$ & $C_{43}$ & $C_{44}$ \\
\hline 1 & 4 & 6 & 2 & 1 \\
\hline 2 & 6 & 5 & 6 & 1 \\
\hline 3 & 14 & 10 & 1 & 1 \\
\hline 4 & 14 & 15 & 1 & 1 \\
\hline 5 & 14 & 15 & 2 & 1 \\
\hline 6 & 14 & 20 & 1 & 1 \\
\hline 7 & 14 & 30 & 1 & 2 \\
\hline 8 & 14 & 30 & 2 & 3 \\
\hline 9 & 14 & 60 & 1 & 2 \\
\hline 10 & 14 & 60 & 2 & 1 \\
\hline 11 & 32 & 30 & 2 & 1 \\
\hline 12 & 54 & 10 & 6 & 1 \\
\hline
\end{tabular}


Let's calculate for the 5th rank the same expressions as for the 4th rank:

$$
\begin{aligned}
& B N(5)=\sum C_{i 4}=23, i=2,4 \\
& B(5)=\sum C_{i 2} \cdot C_{i 4}=522, i=2,4 \\
& R(5)=\sum \frac{C_{i 2} \cdot C_{i 4}}{C_{i 3}}=210, i=2,4 \\
& D(5)=\sum C_{i 1} \cdot C_{i 2} \cdot C_{i 4}=7581, i=2,4 \\
& D C N(5)=\sum\left(2 C_{14}+C_{24}\right)=7 \\
& D C(5)=\sum\left(2 C_{14} \cdot C_{12}+C_{24} \cdot C_{22}\right)=81
\end{aligned}
$$

$$
\begin{aligned}
& \operatorname{DSN}(5)=\sum\left(2 C_{34}+C_{24}\right)=7, i=2,4 \\
& \operatorname{DS}(5)=\sum\left(2 C_{34} \cdot C_{32}+C_{24} \cdot C_{22}\right)=81, i=2,4
\end{aligned}
$$

Summarizing only the lines, where $\mathrm{C}_{42} \mathrm{C}_{43}=5 !=120$. $R N(5)=\sum \frac{C_{i 1} \cdot C_{i 4}}{C_{i 3}}=7, i=2,4$

Summarizing only the lines, where $\mathrm{C}_{42} \mathrm{C}_{43}=5 !=120$. $N S(5)=\sum C_{i 1} \cdot C_{i 2} \cdot C_{i 4}=840, i=2,4$

For the 6 th rank 6 .

Table 7. Blocks containing 2 disjunctive complementary MBFs:

\begin{tabular}{|c|c|c|c|c|}
\hline Nos & $\begin{array}{c}\text { Number of MBFs in the } \\
\text { block }\end{array}$ & Number of isomorphic blocks & Block index & Number of nonisomorphic blocks \\
\hline$C_{10}$ & $C_{11}$ & $C_{12}$ & $C_{13}$ & $C_{14}$ \\
\hline 1 & 8 & 1 & 1 & 1 \\
\hline 2 & 8 & 6 & 1 & 1 \\
\hline 3 & 8 & 10 & 1 & 3 \\
\hline 4 & 8 & 15 & 1 & 3 \\
\hline 5 & 8 & 45 & 2 & 1 \\
\hline 6 & 8 & 60 & 1 & 1 \\
\hline 7 & 8 & 60 & 2 & 1 \\
\hline 8 & 8 & 90 & 1 & 1 \\
\hline 9 & 8 & 90 & 2 & 1 \\
\hline 10 & 8 & 180 & 2 & 1 \\
\hline 11 & 2 & 36 & 1 & 1 \\
\hline 12 & 4 & 10 & 1 & 1 \\
\hline 13 & 4 & 15 & 1 & 1 \\
\hline 14 & 10 & 180 & 1 & 1 \\
\hline 15 & 32 & 90 & 1 & 1 \\
\hline 16 & & & 1 & \\
\hline
\end{tabular}

Table 8. Blocks containing 2 self-dual MBFs:

\begin{tabular}{|c|c|c|c|c|}
\hline Nos & $\begin{array}{c}\text { Number of MBFs in the } \\
\text { block }\end{array}$ & Number of isomorphic blocks & Block index & Number of nonisomorphic blocks \\
\hline$C_{30}$ & $C_{31}$ & $C_{32}$ & $C_{33}$ & $C_{34}$ \\
\hline 1 & 2 & 6 & 2 & 1 \\
\hline 2 & 2 & 10 & 2 & 1 \\
\hline 3 & 2 & 60 & 2 & 1 \\
\hline 4 & 2 & 90 & 1 & 3 \\
\hline 5 & 4 & 6 & 2 & 1 \\
\hline 6 & 6 & 45 & 1 & 1 \\
\hline 7 & 8 & 6 & 1 & 1 \\
\hline 8 & 8 & 20 & 1 & 4 \\
\hline 9 & 8 & 30 & 1 & 2 \\
\hline 10 & 8 & 60 & 2 & 1 \\
\hline 11 & 8 & 180 & 1 & 1 \\
\hline 12 & 10 & 180 & 180 & \\
\hline 13 & & & & 1 \\
\hline
\end{tabular}


Table 9. Cyclic blocks of the 6th rank:

\begin{tabular}{|c|c|c|c|c|}
\hline Nos & $\begin{array}{l}\text { Number of MBFs in the } \\
\text { block }\end{array}$ & Number of isomorphic blocks & Block index & Number of nonisomorphic blocks \\
\hline$C_{40}$ & $C_{41}$ & $C_{42}$ & $C_{43}$ & $C_{44}$ \\
\hline 1 & 4 & 15 & 2 & 1 \\
\hline 2 & 4 & 45 & 4 & 2 \\
\hline 3 & 4 & 60 & 2 & 4 \\
\hline 4 & 4 & 60 & 4 & 2 \\
\hline 5 & 4 & 90 & 2 & 2 \\
\hline 6 & 4 & 90 & 4 & 6 \\
\hline 7 & 4 & 120 & 2 & 2 \\
\hline 8 & 4 & 180 & 2 & 10 \\
\hline 9 & 4 & 180 & 4 & 4 \\
\hline 10 & 4 & 360 & 1 & 1 \\
\hline 11 & 4 & 360 & 2 & 22 \\
\hline 12 & 4 & 720 & 1 & 5 \\
\hline 13 & 6 & 20 & 6 & 2 \\
\hline 14 & 8 & 15 & 2 & 1 \\
\hline 15 & 8 & 45 & 8 & 1 \\
\hline 16 & 8 & 60 & 1 & 1 \\
\hline 17 & 8 & 90 & 2 & 1 \\
\hline 18 & 12 & 30 & 6 & 2 \\
\hline 19 & 12 & 45 & 4 & 3 \\
\hline 20 & 12 & 60 & 2 & 2 \\
\hline 21 & 12 & 90 & 2 & 1 \\
\hline 22 & 12 & 180 & 2 & 3 \\
\hline 23 & 12 & 360 & 1 & 1 \\
\hline 24 & 12 & 360 & 2 & 3 \\
\hline 25 & 12 & 720 & 1 & 1 \\
\hline 26 & 16 & 10 & 2 & 2 \\
\hline 27 & 16 & 15 & 1 & 2 \\
\hline 28 & 16 & 15 & 2 & 1 \\
\hline 29 & 16 & 30 & 1 & 2 \\
\hline 30 & 16 & 45 & 1 & 2 \\
\hline 31 & 16 & 45 & 2 & 12 \\
\hline 32 & 16 & 45 & 4 & 4 \\
\hline 33 & 16 & 60 & 1 & 18 \\
\hline 34 & 16 & 60 & 2 & 11 \\
\hline 35 & 16 & 90 & 1 & 9 \\
\hline 36 & 16 & 90 & 2 & 35 \\
\hline 37 & 16 & 90 & 4 & 1 \\
\hline 38 & 16 & 90 & 8 & 1 \\
\hline 39 & 16 & 120 & 1 & 11 \\
\hline 40 & 16 & 180 & 1 & 53 \\
\hline 41 & 16 & 180 & 2 & 48 \\
\hline 42 & 16 & 180 & 4 & 3 \\
\hline 43 & 16 & 360 & 1 & 118 \\
\hline 44 & 16 & 360 & 2 & 47 \\
\hline 45 & 16 & 720 & 1 & 54 \\
\hline 46 & 20 & 45 & 2 & 2 \\
\hline 47 & 20 & 90 & 2 & 2 \\
\hline 48 & 20 & 180 & 2 & 1 \\
\hline 49 & 20 & 180 & 4 & 2 \\
\hline 50 & 20 & 360 & 2 & 5 \\
\hline 51 & 24 & 30 & 6 & 2 \\
\hline 52 & 24 & 60 & 12 & 2 \\
\hline 53 & 24 & 90 & 2 & 1 \\
\hline 54 & 24 & 90 & 4 & 3 \\
\hline 55 & 24 & 120 & 6 & 2 \\
\hline 56 & 24 & 180 & 2 & 1 \\
\hline 57 & 24 & 180 & 4 & 4 \\
\hline 58 & 24 & 360 & 2 & 2 \\
\hline 59 & 28 & 90 & 2 & 2 \\
\hline 60 & 28 & 180 & 2 & 5 \\
\hline 61 & 28 & 180 & 4 & 1 \\
\hline 62 & 28 & 360 & 2 & 7 \\
\hline
\end{tabular}




\begin{tabular}{|c|c|c|c|c|}
\hline 63 & 28 & 720 & 1 & 1 \\
\hline 64 & 32 & 36 & 2 & 1 \\
\hline 65 & 32 & 90 & 2 & 1 \\
\hline 66 & 32 & 180 & 2 & 2 \\
\hline 67 & 32 & 360 & 1 & 2 \\
\hline 68 & 32 & 360 & 2 & 9 \\
\hline 69 & 36 & 45 & 4 & 2 \\
\hline 70 & 36 & 120 & 6 & 1 \\
\hline 71 & 36 & 180 & 2 & 2 \\
\hline 72 & 36 & 360 & 2 & 6 \\
\hline 73 & 40 & 180 & 2 & 1 \\
\hline 74 & 40 & 360 & 2 & 1 \\
\hline 75 & 44 & 180 & 2 & 1 \\
\hline 76 & 48 & 15 & 6 & 1 \\
\hline 77 & 48 & 30 & 6 & 1 \\
\hline 78 & 48 & 60 & 6 & 1 \\
\hline 79 & 48 & 90 & 8 & 2 \\
\hline 80 & 48 & 120 & 6 & 1 \\
\hline 81 & 48 & 180 & 2 & 5 \\
\hline 82 & 48 & 180 & 4 & 2 \\
\hline 83 & 48 & 240 & 3 & 2 \\
\hline 84 & 48 & 360 & 2 & 1 \\
\hline 85 & 52 & 180 & 2 & 1 \\
\hline 86 & 56 & 180 & 2 & 1 \\
\hline 87 & 56 & 180 & 4 & 2 \\
\hline 88 & 64 & 360 & 2 & 1 \\
\hline 89 & 68 & 360 & 2 & 4 \\
\hline 90 & 70 & 360 & 1 & 1 \\
\hline 91 & 72 & 60 & 12 & 2 \\
\hline 92 & 78 & 90 & 2 & 2 \\
\hline 93 & 80 & 180 & 2 & 2 \\
\hline 94 & 80 & 180 & 4 & 1 \\
\hline 95 & 80 & 360 & 2 & 3 \\
\hline 96 & 84 & 120 & 6 & 2 \\
\hline 97 & 88 & 180 & 4 & 1 \\
\hline 98 & 92 & 180 & 2 & 2 \\
\hline 99 & 96 & 60 & 6 & 1 \\
\hline 100 & 96 & 120 & 6 & 2 \\
\hline 101 & 96 & 180 & 2 & 1 \\
\hline 102 & 104 & 180 & 2 & 1 \\
\hline 103 & 108 & 120 & 6 & 4 \\
\hline 104 & 108 & 180 & 2 & 2 \\
\hline 105 & 112 & 180 & 4 & 1 \\
\hline 106 & 112 & 360 & 2 & 1 \\
\hline 107 & 116 & 360 & 2 & 1 \\
\hline 108 & 120 & 180 & 4 & 2 \\
\hline 109 & 120 & 360 & 2 & 1 \\
\hline 110 & 128 & 90 & 4 & 2 \\
\hline 111 & 128 & 360 & 2 & 1 \\
\hline 112 & 132 & 360 & 2 & 1 \\
\hline 113 & 136 & 180 & 4 & 4 \\
\hline 114 & 140 & 360 & 2 & 2 \\
\hline 115 & 144 & 60 & 6 & 1 \\
\hline 116 & 152 & 180 & 4 & 2 \\
\hline 117 & 152 & 360 & 1 & 1 \\
\hline 118 & 156 & 60 & 12 & 2 \\
\hline 119 & 156 & 360 & 2 & 1 \\
\hline 120 & 162 & 60 & 6 & 2 \\
\hline 121 & 164 & 180 & 2 & 1 \\
\hline 122 & 164 & 180 & 4 & 2 \\
\hline 123 & 172 & 180 & 2 & 1 \\
\hline 124 & 174 & 120 & 6 & 2 \\
\hline 125 & 180 & 180 & 2 & 1 \\
\hline 126 & 184 & 180 & 2 & 2 \\
\hline 127 & 184 & 360 & 1 & 1 \\
\hline 128 & 188 & 180 & 2 & 1 \\
\hline 129 & 196 & 360 & 2 & 1 \\
\hline
\end{tabular}




\begin{tabular}{|c|c|c|c|c|}
\hline 130 & 204 & 60 & 12 & 2 \\
\hline 131 & 204 & 120 & 6 & 1 \\
\hline 132 & 204 & 180 & 2 & 1 \\
\hline 133 & 204 & 360 & 2 & 1 \\
\hline 134 & 208 & 180 & 2 & 2 \\
\hline 135 & 212 & 180 & 2 & 1 \\
\hline 136 & 216 & 180 & 4 & 1 \\
\hline 137 & 240 & 120 & 3 & 1 \\
\hline 138 & 244 & 360 & 2 & 1 \\
\hline 139 & 248 & 90 & 4 & 2 \\
\hline 140 & 248 & 90 & 8 & 2 \\
\hline 141 & 256 & 90 & 2 & 1 \\
\hline 142 & 256 & 180 & 2 & 1 \\
\hline 143 & 256 & 360 & 1 & 1 \\
\hline 144 & 264 & 120 & 6 & 1 \\
\hline 145 & 264 & 360 & 2 & 1 \\
\hline 146 & 268 & 180 & 2 & 1 \\
\hline 147 & 288 & 120 & 6 & 1 \\
\hline 148 & 296 & 180 & 4 & 1 \\
\hline 149 & 336 & 90 & 4 & 2 \\
\hline 150 & 376 & 90 & 4 & 1 \\
\hline 151 & 376 & 180 & 2 & 1 \\
\hline 152 & 396 & 120 & 6 & 1 \\
\hline 153 & 408 & 180 & 4 & 1 \\
\hline 154 & 432 & 20 & 6 & 1 \\
\hline 155 & 432 & 60 & 6 & 2 \\
\hline 156 & 432 & 90 & 2 & 1 \\
\hline 157 & 438 & 120 & 6 & 2 \\
\hline 158 & 444 & 120 & 6 & 1 \\
\hline 159 & 456 & 120 & 6 & 1 \\
\hline 160 & 468 & 120 & 6 & 1 \\
\hline 161 & 504 & 120 & 6 & 1 \\
\hline 162 & 512 & 90 & 8 & 2 \\
\hline 163 & 540 & 120 & 6 & 1 \\
\hline 164 & 576 & 120 & 6 & 1 \\
\hline 165 & 696 & 120 & 6 & 1 \\
\hline 166 & 732 & 180 & 4 & 2 \\
\hline 167 & 760 & 180 & 4 & 1 \\
\hline 168 & 768 & 120 & 6 & 1 \\
\hline 169 & 792 & 120 & 6 & 1 \\
\hline 170 & 828 & 120 & 6 & 1 \\
\hline 171 & 1232 & 90 & 8 & 1 \\
\hline 172 & 2052 & 120 & 6 & 1 \\
\hline 173 & 2064 & 120 & 6 & 1 \\
\hline
\end{tabular}

Let's calculate for the 6th rank the same expressions as for the 4th one:

$$
\begin{aligned}
& B N(6)=\sum C_{i 4}=775, i=1,3,4 \\
& B(6)=\sum C_{i 2} \cdot C_{i 4}=189182, i=1,3,4 \\
& R(6)=\sum \frac{C_{i 2} \cdot C_{i 4}}{C_{i 3}}=16353, i=1,3,4 \\
& D(6)=\sum C_{i 1} \cdot C_{i 2} \cdot C_{i 4}=7828354, i=1,3,4 \\
& D C N(6)=\sum\left(2 C_{14}+C_{24}\right)=39, \\
& D C(6)=\sum\left(2 C_{14} \cdot C_{12}+C_{24} \cdot C_{22}\right)=2646 \\
& D S N(6)=\sum\left(2 C_{34}+C_{24}\right)=30, i=1,3,4 \\
& D S(6)=\sum\left(2 C_{34} \cdot C_{32}+C_{24} \cdot C_{22}\right)=2646, i=1,3,4
\end{aligned}
$$

Summarizing only the lines, where C42C43 $=6 !=720$.
$R N(6)=\sum \frac{C_{i 1} \cdot C_{i 4}}{C_{i 3}}=7281, i=1,3,4$

Summarizing only the lines where $\mathrm{C} 42 \mathrm{C} 43=6 !=720$. $N S(6)=\sum C_{i 1} \cdot C_{i 2} \cdot C_{i 4}=5242320, i=1,3,4$

This method is not limited by the number of variables because for each MBF with any number of variables $n$ we can build a block containing this MBF using operations of disjunctive complement and duality. In this case, it is sufficient to build only nonisomorphic blocks to describe all MBFs of $n$ variables. This significantly facilitates the description of all MBFs of $n$ variables. For example, all MBFs of 6 variables can be split into 189182 blocks but only 775 of them can be selected as mutually nonisomorphic. Classes of equivalence and nonisomorphic blocks are directly connected with the Dedekind numbers. 


\section{REFERENCES}

[1] Dedekind R. Über Zerlegungen von Zahlen durch ihre grössten gemeinsamen Teiler. Festschrift Hoch. Braunschweig u. ges. Werke. II, 1897, 103-148.

[2] Wiedemann, Doug A computation of the eighth Dedekind number. Order 8 (1991), no. 1, 5-6.

[3] Tkachenco V.G., Sinyavsky O.V. (2016). Blocks of Monotone Boolean Functions, Computer Science and Information Technology Vol. 4(2), pp. 72 - 78. DOI: 10.13189/csit.2016.040203.

[4] Tkachenco V.G., Sinyavsky O.V. (2016). Blocks of Monotone Boolean Functions of Rank 5, Computer Science and Information Technology 4(4): pp.139-146, 2016, DOI: 10.13189/csit.2016.040402

[5] Ткаченко В.Г. Метод построения блоков монотонных булевых функций. Наукові праці ОНАЗ ім. О.С. Попова. 2011. № 1. C.79 - 83.

[6] Ткаченко В.Г, Иваницкий А.М. Взаимосвязь между матроидами и монотонными булевыми функциями электрических цепей. Наукові праці ОНАЗ ім. О.С. Попова. 2009. № 1. С.18-26.

[7] T. Stephen, T. Yusun, Counting inequivalent monotone boolean functions, Discrete Appl. Math. 167 (2014) 15-24. doi:10.1016/j.dam.2013.11.015. URL http://dx.doi.org/10.10 16/j.dam.2013.11.015 